\title{
Single-gap versus two-gap scenario: Specific heat and thermodynamic critical field of the noncentrosymmetric superconductor BeAu
}

\author{
Rustem Khasanov $\odot,{ }^{1, *}$ Ritu Gupta $\odot,{ }^{1}$ Debarchan Das $\odot,{ }^{1}$ Andreas Leithe-Jasper, ${ }^{2}$ and Eteri Svanidze ${ }^{2}$ \\ ${ }^{1}$ Laboratory for Muon Spin Spectroscopy, Paul Scherrer Institute, 5232 Villigen, Switzerland \\ ${ }^{2}$ Max-Planck-Institut für Chemische Physik fester Stoffe, Nöthnitzer Straße 40, 01187 Dresden, Germany
}

(Received 25 April 2020; revised 8 July 2020; accepted 10 July 2020; published 27 July 2020)

\begin{abstract}
Temperature evolutions of the electronic specific heat $C_{\mathrm{e}}(T)$ [A. Amon et al., Phys. Rev. B 97, 014501 (2018)] and the thermodynamic critical field $B_{\mathrm{c}}(T)$ [R. Khasanov et al., Phys. Rev. Research 2, 023142 (2020)] of the $\mathrm{BeAu}$ superconductor were reconsidered within the framework of the single-gap and two-gap scenarios. The analysis shows that the single-gap approach, by being able to describe satisfactorily the temperature dependence of $C_{\mathrm{e}}(T)$, fails in the case of $B_{\mathrm{c}}(T)$. The self-consistent two-gap model, in contrast, is able to describe both thermodynamic quantities by using a similar set of parameters. Our results imply that in some particular cases, such as in the case of $\mathrm{BeAu}$, measurement of one single thermodynamic quantity may not be enough in order to speculate on the nature of the superconducting pairing mechanism.
\end{abstract}

DOI: 10.1103/PhysRevB.102.014514

\section{INTRODUCTION}

Measurements of temperature dependences of thermodynamic quantities such as the superfluid density $\rho_{s}$, the electronic specific heat $C_{e}$, and the thermodynamic critical field $B_{\mathrm{c}}$ are among the primary ways used to test pairing mechanisms of new superconductors [1-6]. In particular, such experiments allow us (i) to distinguish between the single-band and multiband scenarios [7-14], (ii) to determine the superconducting gap structure [15-26], (iii) to calculate the temperature evolution(s) of the superconducting energy gaps [27-33], etc. Obviously, the parameters obtained from the analysis of various thermodynamic quantities need to be consistent with each other. In other words, the temperature evolution of the superfluid density, the specific heat, and the thermodynamic critical field should be described within a similar model by using the same set of parameters. For example, this is the case in the analysis of conventional elemental and binary superconductors within the framework of the empirical $\alpha$ model (see Refs. [34-38]) and the Eliashberg approach (see the classic reviews of Carbotte [39] and Marsiglio and Carbotte [40]) and the two-gap superconductors within the self-consistent approach using models developed by Kogan et al. [41,42] and Bussmann-Holder et al. [43-45].

Our interest in interpreting the superfluid density, the specific heat, and the thermodynamic critical field within the single-gap and two-gap scenarios was initiated by recent publications pointing to contradictory results for the noncentrosymmetric $\mathrm{BeAu}$ superconducting compound [46-50]. Indeed, Refs. [46-49] consider BeAu to host a single isotropic gap, which is a characteristic of predominantly $s$-wave spinsinglet pairing in the weak-coupling limit. Such evidence comes from the electronic specific heat measurements, suggesting that $C_{\mathrm{e}}(T)$ is well described within the single-gap

*rustem.khasanov@psi.ch approach with the ratio $\alpha=\Delta(0) / k_{\mathrm{B}} T_{\mathrm{c}}$ ranging from the weakly coupled $\mathrm{BCS}$ value $\alpha_{\mathrm{BCS}}=1.764$ up to $\alpha \simeq$ 1.88 [46-49]. Here, $\Delta(0), T_{\mathrm{c}}$, and $k_{\mathrm{B}}$ are the zero-temperature value of the gap, the superconducting transition temperature, and the Boltzmann constant, respectively. On the other hand, precise measurements of the thermodynamic critical field reported in Ref. [50] show that the temperature evolution of $B_{\mathrm{c}}$ cannot be described within the single-gap scenario and the presence of at least two different types of the superconducting order parameters is required. The analysis of $B_{\mathrm{c}}(T)$ within the self-consistent two-gap approach suggests the presence of two superconducting energy gaps with the ratios $\Delta / k_{\mathrm{B}} T_{\mathrm{c}} \simeq 2.26$ and 1.19 for the big and small gaps, respectively.

The aim of the present paper is to resolve the above contradiction in interpreting the $C_{\mathrm{e}}(T)$ and $B_{\mathrm{c}}(T)$ data of $\mathrm{BeAu}$. In order to solve this dilemma, both $C_{\mathrm{e}}(T)$ and $B_{\mathrm{c}}(T)$, as they were reported in Refs. [47,50], respectively, were analyzed by means of the single-gap and two-gap models. Our result confirms that the two-gap approach allows us to describe both thermodynamic quantities with the same set of parameters. Moreover, the fact that $C_{\mathrm{e}}(T)$ could be interpreted within the single-gap scenario becomes an interesting feature of the $\mathrm{BeAu}$ superconductor. In $\mathrm{BeAu}$, two contributions to the electronic specific heat, by being summed together, result in a behavior which is indistinguishable from expectations of the single-gap scenario. The simulations show that this is also the case for the temperature evolution of the superfluid density. Our results imply, therefore, that in some particular cases, such as in the case of BeAu studied here, measurement of one single thermodynamic quantity may not be enough in order to speculate about the mechanism of superconductivity. The validity of the model used to describe the experimental data for one particular thermodynamic quantity needs to be cross-checked by analyzing the other quantities as well.

This paper is organized as follows: The analyses of the $C_{\mathrm{e}}(T)$ and $B_{\mathrm{c}}(T)$ dependences of BeAu within the single-gap and two-gap scenarios are presented in Secs. II A and II B, 
respectively. In Sec. III A, the specific heat and the superfluid density data, simulated by means of the two-gap model, are compared with the one-gap model fits. The simultaneous single-gap and multiple-gap responses of other superconducting materials are discussed in Sec. III B. Conclusions follow in Sec. IV. Descriptions of the single-gap and two-gap approaches for analyzing the specific heat, the thermodynamic critical field, and the superfluid density are given in the Appendix.

\section{THE SPECIFIC HEAT AND THE THERMODYNAMIC CRITICAL FIELD OF BeAu}

In this section, the comparison of the single-gap and twogap approaches in analyzing the temperature evolution of the specific heat $C_{\mathrm{e}}(T)$ and the thermodynamic critical field $B_{\mathrm{c}}(T)$ of the BeAu superconductor is discussed. The $C_{\mathrm{e}}(T)$ and $B_{\mathrm{c}}(T)$ data sets were taken from Refs. [47,50], respectively. Note that currently, three sets of $C_{\mathrm{e}}(T)$ and $B_{\mathrm{c}}(T)$ data for $\mathrm{BeAu}$ are available in the literature [46-51]. Among them, the $C_{\mathrm{e}}(T)$ and $B_{\mathrm{c}}(T)$ dependences from Refs. [47,50] were measured on BeAu samples from the same batch. For this reason, these two data sets are going to be compared in this paper. Note that the comparison of $B_{\mathrm{c}}(T)$ from Ref. [50] with $C_{\mathrm{e}}(T)$ curves from Refs. [46,48] leads to the same basic conclusions (not shown).

\section{A. $C_{\mathrm{e}}(T)$ and $B_{\mathrm{c}}(T)$ of BeAu: Single-gap scenario}

The single-gap analysis of the specific heat $C_{\mathrm{e}}(T)$ and the thermodynamic critical field $B_{\mathrm{c}}(T)$ data of the BeAu superconductor followed the procedure described in Appendix Sec. A 1. Within the single-gap scenario, Eqs. (A1) and (A3) were fit to the $C_{\mathrm{e}}(T)$ and $B_{\mathrm{c}}(T)$ data sets, respectively. The temperature dependence of the superconducting energy gap was assumed to be the same and was described within the $\alpha$ model (Sec. A 1 b) by using

$$
\Delta(T)=\Delta(0) \delta_{\mathrm{BCS}}(T)=\alpha k_{\mathrm{B}} T_{\mathrm{c}} \delta_{\mathrm{BCS}}(T) .
$$

Here, $\Delta(0)$ is the zero-temperature value of the gap, $\delta_{\mathrm{BCS}}(T)$ is the normalized superconducting energy gap with the temperature evolution following the BCS theory (see Ref. [52], Sec. A 1 b, and Fig. 7), and $\alpha=\Delta(0) / k_{\mathrm{B}} T_{\mathrm{c}}$ is the parameter accounting for the deviation from the weakly coupled BCS prediction $\left(\alpha_{\mathrm{BCS}} \simeq 1.764\right)$.

The results of the analysis are shown by solid black lines in Figs. 1 and 2 for $C_{\mathrm{e}}(T)$ and $B_{\mathrm{c}}(T)$ data, respectively. Note that the thermodynamic critical field data from Ref. [50] are expressed in terms of the deviation function $D(T)=B_{\mathrm{c}}(T)-$ $B_{\mathrm{c}}(0)\left[1-T^{2} / T_{\mathrm{c}}^{2}\right]$, which represents the deviation of $B_{\mathrm{c}}(T)$ from the parabolic type of behavior.

The analysis of the specific heat data itself agrees with the experiment (Fig. 1). The fit parameters are $T_{\mathrm{c}}=3.29(1) \mathrm{K}$, $\gamma_{\mathrm{n}}=1.813(5) \mathrm{mJ} / \mathrm{mol} \mathrm{K}^{2}$, and $\alpha=\Delta(0) / k_{\mathrm{B}} T_{\mathrm{c}}=1.686(8)$. The goodness of the fit could be further judged from the calculated $\chi_{\text {norm }}^{2}$ parameter (the sum of the mean-square deviations divided by number of degrees of freedom minus 1), which for a "good fit" should not be far from unity. The value of $\chi_{\text {norm }}^{2}=1.87$ is found, thus suggesting satisfactory agreement between the data and the fit. It is worth noting here that

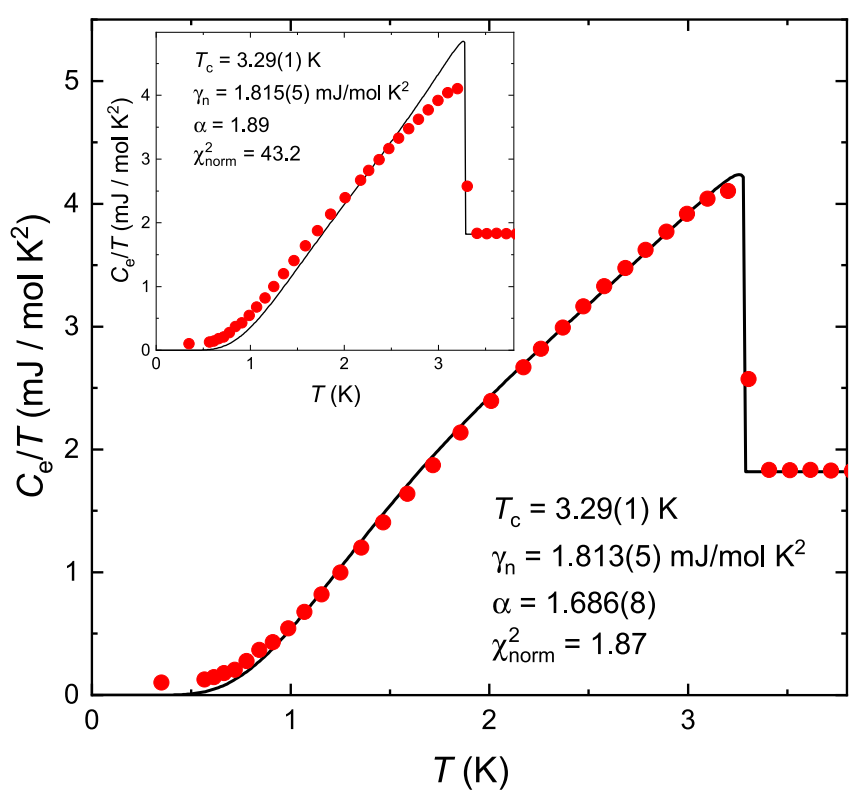

FIG. 1. The electronic specific heat $C_{\mathrm{e}}(T)$ of $\mathrm{BeAu}$ (after Ref. [47]). The solid line is the fit of the single-gap $\alpha$ model to the experimental data with $\alpha=\Delta(0) / k_{\mathrm{B}} T_{\mathrm{c}}=1.686(8)$. Satisfactory agreement between the fit and the data is confirmed by the value of $\chi_{\text {norm }}^{2}=1.87$. The inset shows the results of the fit by fixing the value of the parameter $\alpha=1.89$ to that obtained in the analysis of $B_{\mathrm{c}}(T)$ data. See text for details.

the value of $\alpha=1.686(8)$ is only slightly smaller than the weakly coupled BCS value $\alpha_{\mathrm{BCS}}=1.764$. This agrees with the previous observations suggesting the presence of a single isotropic energy gap in BeAu [46-49].

The analysis of $B_{\mathrm{c}}(T)$ data does not lead to agreement between the theory and the experiment (Fig. 2). The theoretical $D(T)$ curve deviates strongly from the data. The value $\chi_{\text {norm }}^{2}=8.82$ remains far from unity. The best-fit parameters are $T_{\mathrm{c}}=3.195(3) \mathrm{K}, B_{\mathrm{c}}(0)=26.65(6) \mathrm{mT}$, and $\alpha=1.89(1)$.

For comparison, the $C_{\mathrm{e}}(T)\left[B_{\mathrm{c}}(T)\right]$ data were fit by fixing the parameter $\alpha$ to the value obtained in the analysis of the critical field (specific heat) data. The results of the fits are shown in insets of Figs. 1 and 2 for $C_{\mathrm{e}}(T)$ and $B_{\mathrm{c}}(T)$, respectively. The agreement between the fit and the data is relatively poor, which is also confirmed by high values of $\chi_{\text {norm }}^{2}\left[\chi_{\text {norm }}^{2}=43.2\right.$ for $C_{\mathrm{e}}(T)$ and $\chi_{\text {norm }}^{2}=64.9$ for $\left.B_{\mathrm{c}}(T)\right]$.

\section{B. $C_{\mathrm{e}}(T)$ and $B_{\mathrm{c}}(T)$ of BeAu: Two-gap scenario}

The two-gap analysis followed the procedure described in subsection 2 of the Appendix. Equations (A10) and (A12) were fit simultaneously to the $C_{\mathrm{e}}(T)$ and $B_{\mathrm{c}}(T)$ experimental data. The individual components of these equations were described by Eqs. (A1), (A4), and (A5), respectively. The temperature dependences of the big $\left[\Delta_{1}(T)\right]$ and small $\left[\Delta_{2}(T)\right]$ superconducting energy gaps were obtained by solving the system of coupled nonlinear equations [Eq. (A14)].

At first glance, the number of fit parameters is quite high, so the fitting procedure might be unstable, and good fits are expected to be found for various combinations of the parameters. The number of fit parameters is seven for Eq. (A14) $\left(N_{1}, N_{2}, V_{11}, V_{12}, V_{21}, V_{22}\right.$, and $\left.\omega_{\mathrm{D}}\right)$, four for Eq. (A10) $\left(T_{\mathrm{c}}, \gamma_{\mathrm{n}}\right.$, 


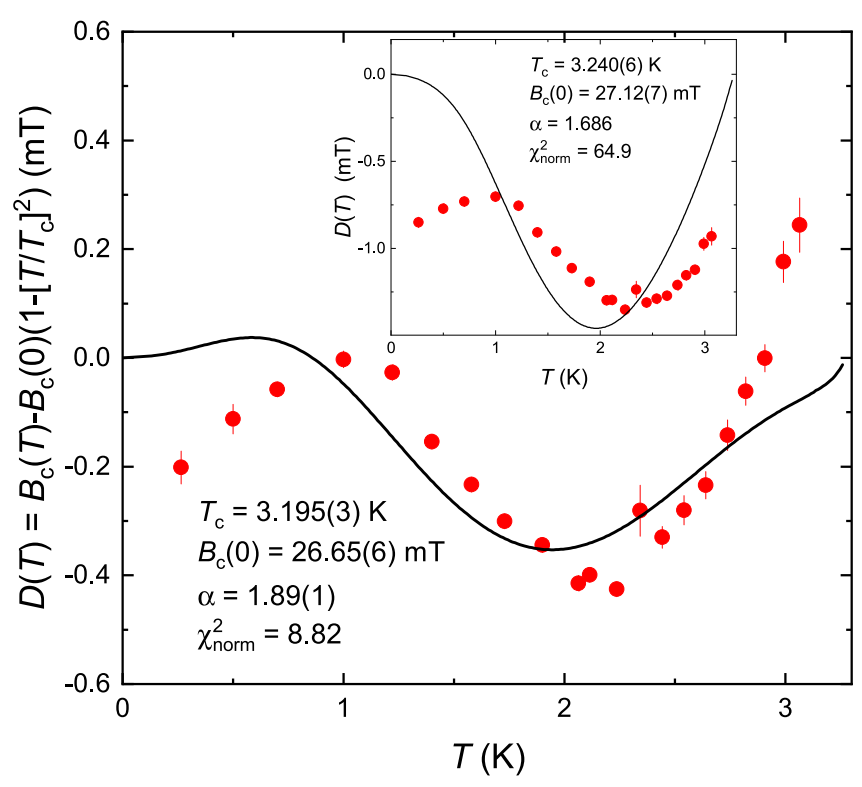

FIG. 2. The deviation of the temperature evolution of the thermodynamic critical field $B_{\mathrm{c}}(T)$ of BeAu from the parabolic behavior $D(T)=B_{\mathrm{c}}(T)-B_{\mathrm{c}}(0)\left[1-T^{2} / T_{\mathrm{c}}^{2}\right]$ (after Ref. [50]). The solid line is the fit of the single-gap $\alpha$ model to the experimental data with $\alpha=\Delta(0) / k_{\mathrm{B}} T_{\mathrm{c}}=1.89(1)$. The inset shows the results of the fit by fixing the value of the parameter $\alpha=1.686$ to that obtained in the analysis of $C_{\mathrm{e}}(T)$ data. See text for details.

$\gamma_{\mathrm{n} 1}$, and $\gamma_{\mathrm{n} 2}$ ), and five for Eq. (A12) $\left[T_{\mathrm{c}}, B_{\mathrm{c}}(0), \gamma_{\mathrm{n}}, \gamma_{\mathrm{n} 1}\right.$, and $\left.\gamma_{\mathrm{n} 2}\right]$. This results in a total of 16 fit parameters. In reality, the number of fit parameters could be reduced substantially since some of them are related to each other, while the others could be determined in an independent set of experiments.

The following arguments can be considered:

(i) Within the free-electron approximation, the product $\gamma_{\mathrm{n} i} / \gamma_{\mathrm{n}}(i=1,2)$ from Eqs. (A10) and (A12) is equal to the corresponding partial density of states $N_{i}$ in Eq. (A14), so that $\gamma_{\mathrm{n} 1} / \gamma_{\mathrm{n}} \equiv N_{1}$ and $\gamma_{\mathrm{n} 2} / \gamma_{\mathrm{n}} \equiv N_{2}$.

(ii) The value of the Debye frequency could be independently derived from the specific heat measurements. Amon et al. [47] found $\omega_{\mathrm{D}} \simeq 33.4 \mathrm{meV}$. Note that slightly smaller $\omega_{\mathrm{D}} \simeq 25.4 \mathrm{meV}$ was reported by Rebar et al. $[46,48]$.

(iii) The value of the electronic specific heat component (Sommerfeld constant) could be determined from the linear fit of $C_{\mathrm{e}}(T)$ data for $T$ above $T_{\mathrm{c}}$ (see Fig. 3). The fit reveals $\gamma_{\mathrm{n}} \simeq 1.81 \mathrm{~mJ} / \mathrm{mol} \mathrm{K}^{2}$.

(iv) The electronic specific heat component $\gamma_{\mathrm{n}}$ is derived as the sum of two contributions, $\gamma_{\mathrm{n}}=\gamma_{\mathrm{n} 1}+\gamma_{\mathrm{n} 2}$ [7].

By considering the above arguments, the total number of fitting parameters for simultaneous analysis of $C_{\mathrm{e}}(T)$ and $B_{\mathrm{c}}(T)$ data is reduced from 16 to 8 , namely, four coupling constants (two intraband, $V_{11}$ and $V_{22}$, and two interband, $V_{12}$ and $V_{21}$, coupling strengths), two transition temperatures $\left(T_{\mathrm{c}}^{C_{\mathrm{e}}}\right.$ and $T_{\mathrm{c}}^{B_{\mathrm{c}}}$ ) [53], the zero-temperature value of the thermodynamic critical field $B_{\mathrm{c}}(0)$, and the weighting factor $w_{C_{\mathrm{e}}} \equiv$ $w_{F_{\mathrm{s}}}=\gamma_{\mathrm{n} 1} / \gamma_{\mathrm{n}}$.

The analysis of $C_{\mathrm{e}}(T)$ and $B_{\mathrm{c}}(T)$ data within the two-gap scenario was performed in a few steps. First, by solving two nonlinear coupled gap equations [Eq. (A14)], the tempera-
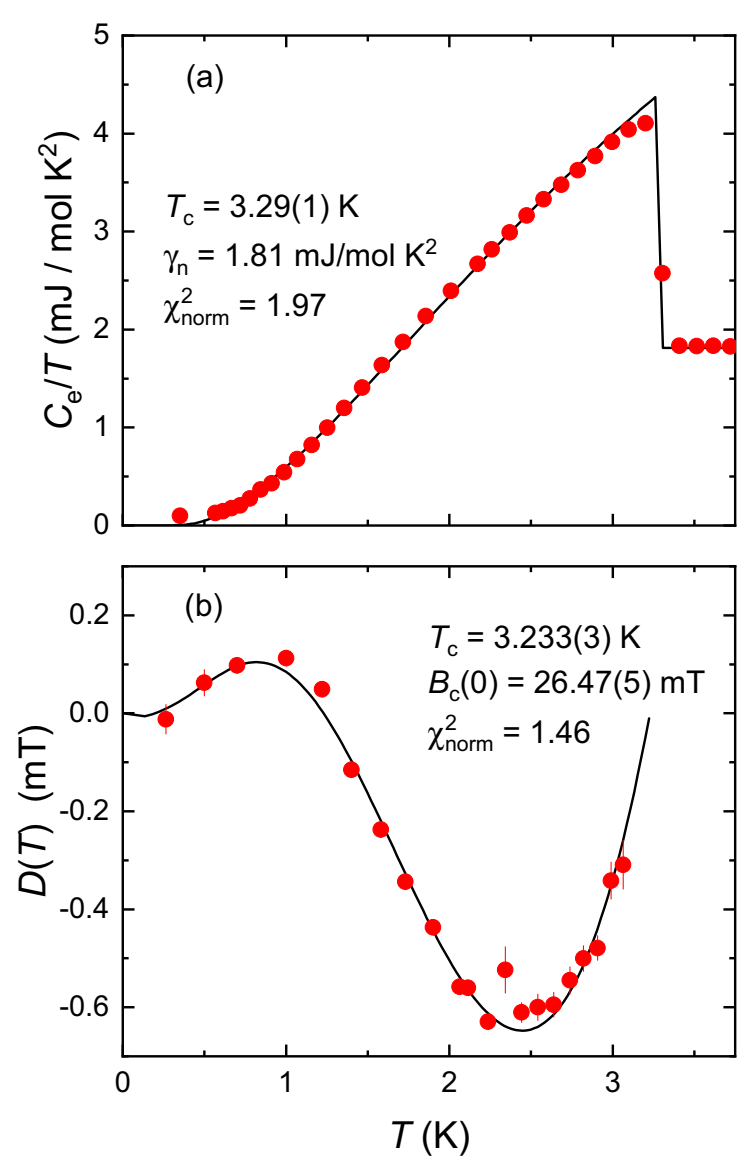

FIG. 3. (a) The electronic specific heat $C_{\mathrm{e}}(T)$ of BeAu (after Ref. [47]) and (b) the deviation of the temperature evolution of the thermodynamic critical field $B_{\mathrm{c}}(T)$ of BeAu from the parabolic behavior $D(T)=B_{\mathrm{c}}(T)-B_{\mathrm{c}}(0)\left[1-T^{2} / T_{\mathrm{c}}^{2}\right]$ (after Ref. [50]) analyzed within the self-consistent two-gap model. Equations (A10) and (A12) were fitted simultaneously to the data with the same big $\Delta_{1}(T)$ and small $\Delta_{2}(T)$ superconducting energy gaps and the weighting factor $w_{C_{\mathrm{e}}} \equiv w_{F_{\mathrm{s}}}=0.63$. See text for details.

ture dependences of the big and small superconducting gaps were calculated. Note that the combination of the coupling strengths and the Debye frequency determines uniquely the value of $T_{\mathrm{c}}$. By substituting $\Delta_{1}(T)$ and $\Delta_{2}(T)$ into Eqs. (A10) and (A12), the corresponding $C_{\mathrm{e}}(T)$ and $B_{\mathrm{c}}(T)$ were derived. The difference between the experimental data and the theory was minimized by adjusting $T_{\mathrm{c}}^{C_{\mathrm{e}}}, T_{\mathrm{c}}^{B_{\mathrm{c}}}, B_{\mathrm{c}}(0)$, and $w_{C_{\mathrm{e}}} \equiv w_{F_{\mathrm{s}}}$. The tiny difference between $T_{\mathrm{c}}^{C_{\mathrm{e}}}$ and $T_{\mathrm{c}}^{B_{\mathrm{c}}}$ was accounted for by assuming $T_{\mathrm{c}}^{C_{\mathrm{e}}}=$ coeff $\times T_{\mathrm{c}}^{B_{\mathrm{c}}}($ coeff $\simeq 0.983$, see Ref. [53]), which is equal to rescaling both gaps as $\Delta_{1,2}(T /$ coeff). In the next step, the coupling strength parameters were readjusted, and the calculations of $C_{\mathrm{e}}(T)$ and $B_{\mathrm{c}}(T)$ were repeated. After about 10 to 15 such iterations the fit converges.

The results of the self-consistent two-gap analysis are presented in Figs. 3(a) and 3(b) for the $C_{\mathrm{e}}(T)$ and $B_{\mathrm{c}}(T)$ data sets, respectively. The fit parameters are $B_{\mathrm{c}}(0)=26.47(5) \mathrm{mT}, w_{C_{\mathrm{e}}}=0.630(3), V_{11}=0.243(3)$, $V_{22}=0.319(4), V_{12}=0.281(3)$, and $V_{21}=0.068(1)$. The values of the superconducting transition temperature are $T_{\mathrm{c}}^{C_{\mathrm{e}}}=3.29(1) \mathrm{K}$ and $T_{\mathrm{c}}^{B_{\mathrm{c}}}=3.233(3) \mathrm{K}$. Obviously, the selfconsistent two-gap model describes both sets of experimental 


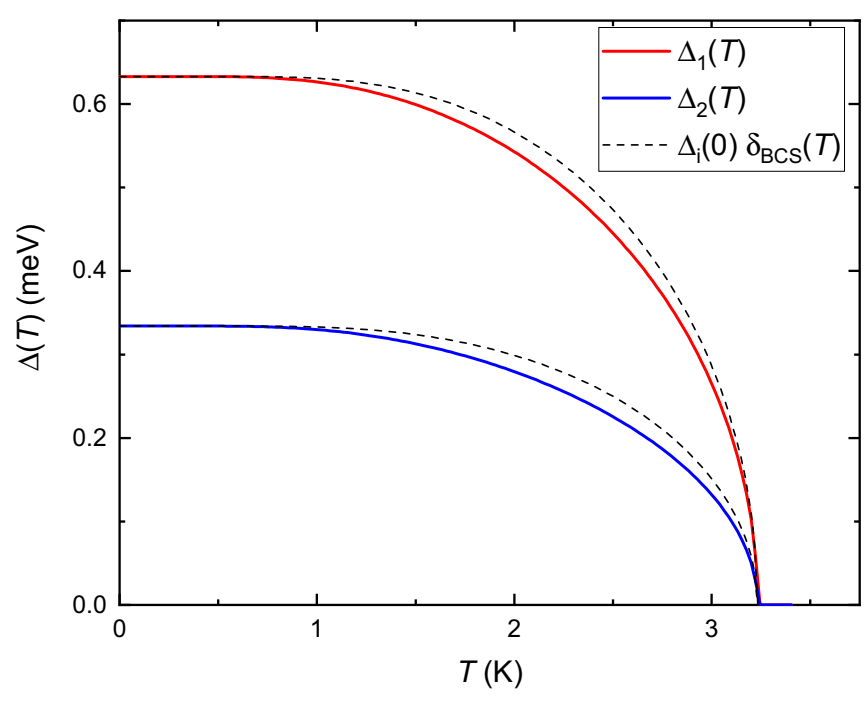

FIG. 4. Temperature evolution of the big ( $\Delta_{1}$, red line) and small $\left(\Delta_{2}\right.$, blue line) superconducting energy gaps, obtained within the framework of the self-consistent two-gap model. The dashed lines are the weakly coupled BCS prediction [52].

data remarkably well. The values of $\chi_{\text {norm }}^{2}$ are found to be 1.97 and 1.46 for the $C_{\mathrm{e}}(T)$ and $B_{\mathrm{c}}(T)$ data sets, respectively. A substantial improvement of $\chi_{\text {norm }}^{2}$ [from 8.82 to 1.46 ; see Figs. 2 and 3(b)] is observed for the $B_{\mathrm{c}}(T)$ set of data. The slight increase in $\chi_{\text {norm }}^{2}$ for $C_{\mathrm{e}}(T)$ [from 1.87 to 1.97 ; see Figs. 1 and 3(a)] is most likely caused by the increased number of fit parameters in the two-gap model compared to the single-gap one.

The temperature evolution of the big and small gaps and the comparison of their temperature dependences with the weakly coupled BCS prediction (see Fig. 7) are presented in Fig. 4. Obviously, both $\Delta_{1}(T)$ and $\Delta_{2}(T)$ are slightly weaker than the expectation of the weakly coupled BCS model (dashed lines). Such behavior is expected in the case of multiband superconductors. It is confirmed theoretically as well as experimentally for the most famous two-gap superconductor $\mathrm{MgB}_{2}$ [32,41,54-56].

\section{COMPARISON BETWEEN THE SINGLE-GAP AND TWO-GAP APPROACHES}

\section{A. The specific heat and the superfluid density data}

An obvious question arises: Why was the previous analysis of the specific heat data of the BeAu superconductor found to be consistent with the presence of a single isotropic energy gap [46-49]? In order to answer this question, the simulated "two-gap" $C_{\mathrm{e}}(T)$ curve [see Fig. 3(a)] was reanalyzed by using the single-gap $\alpha$ model [see Eq. (A1) and Fig. 5]. In addition, the superfluid density $\rho_{\mathrm{s}}(T) / \rho_{\mathrm{s}}(0)$ curve was calculated by means of Eq. (A11) and further reanalyzed within the single-gap approach by using Eq. (A2) (see Fig. 6). In the two-gap $\rho_{\mathrm{s}}$ calculation, the "weighting" parameter $w_{\rho}$ was assumed to be equal to $w_{C_{\mathrm{e}}}$. Surprisingly, in both cases the single-gap curves stay relatively close to the two-gap ones. The fit parameters are $\alpha=1.734(5)$ and 1.675(6) for the specific heat and the superfluid density, respectively. Note that
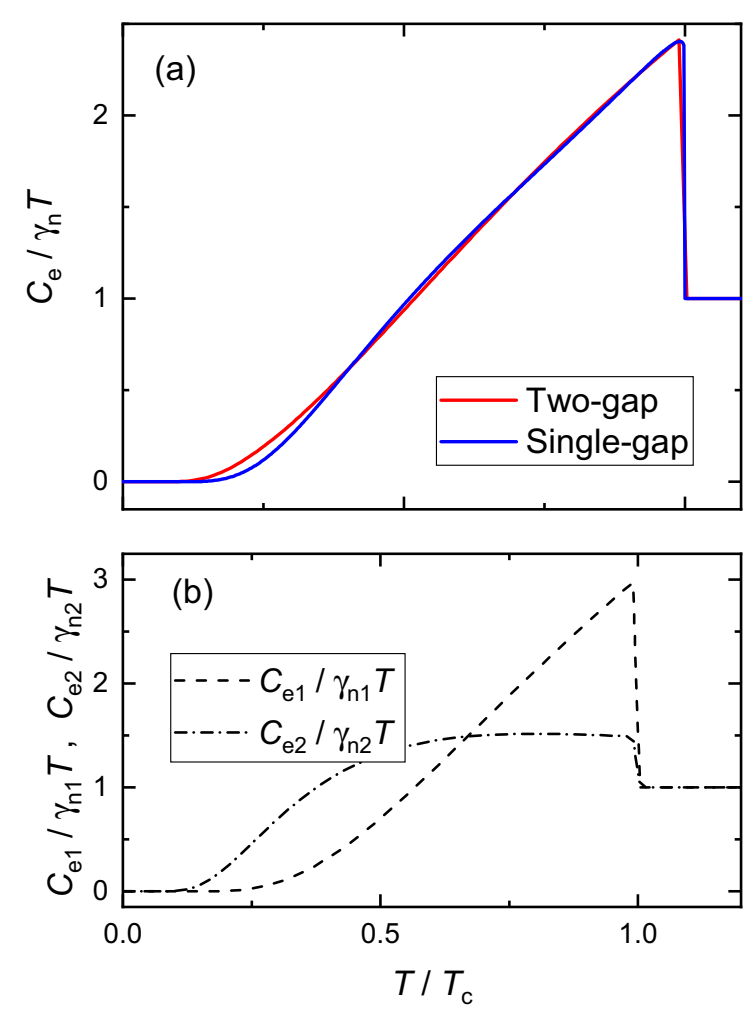

FIG. 5. (a) Comparison of the temperature evolution of the electronic specific heat obtained within the framework of the selfconsistent two-gap model (red line) with the fit to the single-gap $\alpha$ model [Eq. (A1), blue line]. (b) The individual contributions to the specific heat, obtained within the framework of the self-consistent two-gap model. See text for details.

$\alpha=1.734$, obtained from the fit of two-gap specific heat data, stays relatively close to the values reported in Refs. [46-49].

This is rather unexpected, meaning that in the particular case of the BeAu superconductor, the summed contributions of the big and small gaps [dashed and dash-dotted lines in Figs. 5(b) and 6(b)] are indistinguishable from the single-gap scenario. By following the data presented in Figs. 5 and 6, this becomes true for at least two thermodynamical quantities, such as the electronic specific heat and the superfluid density. On the other hand, the thermodynamic critical field $B_{\mathrm{c}}(T)$ fails to be described within the single-gap behavior and requires the use of the self-consistent two-gap model for its description (see also Ref. [50]).

\section{B. Other superconducting materials}

The situation described in our paper is not unique for the particular $\mathrm{BeAu}$ superconductor. For example, the $\mathrm{LaFeAs}_{1-x} \mathrm{Fe}_{x}, \mathrm{SrPt}_{3} \mathrm{P}$, and $\mathrm{Nb}_{5} \mathrm{Ir}_{3-x} \mathrm{Pt}_{x} \mathrm{O}$ superconducting compounds can be mentioned. In $\mathrm{LaFeAs}_{1-x} \mathrm{Fe}_{x}$, the superfluid density data are well described within the single $s$-wave gap approach [57], while the Andreev reflection studies point to the presence of several (at least two) superconducting energy gaps with different absolute values [58]. In $\mathrm{SrPt}_{3} \mathrm{P}$, the single-gap behavior of the superfluid density was found to be in contradiction to the multiple-band response of the upper critical field [59]. In that paper it was also shown that two 

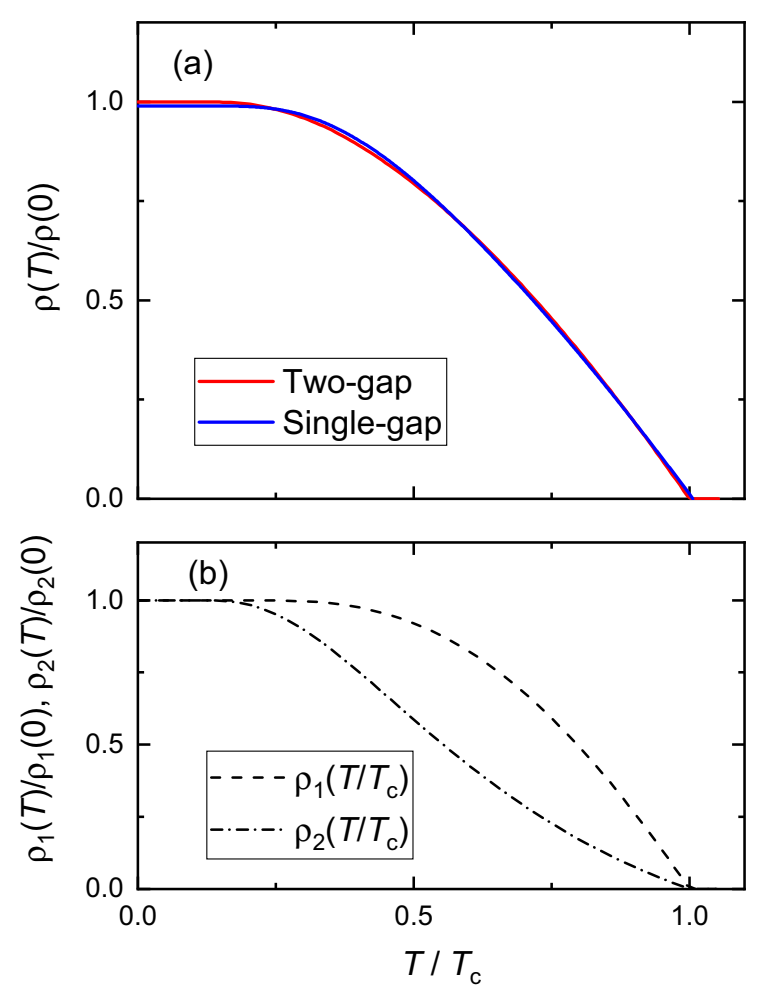

FIG. 6. (a) Comparison of the temperature evolution of the superfluid density $\rho_{\mathrm{s}}(T) / \rho_{s}(0)$ obtained within the self-consistent two-gap model [Eq. (A11), red line] with the fit to the single-gap $\alpha$ model [Eq. (A2), blue line]. (b) The individual contributions to the superfluid density, obtained within the framework of the selfconsistent two-gap model. See text for details.

superconducting energy gaps with different values may, in principle, result in temperature evolution of the superfluid density which is indistinguishable from the single-gap approach (see the discussion in Sec. S3b in the Supplemental Material of Ref. [59]). In the $\mathrm{Nb}_{5} \mathrm{Ir}_{3-x} \mathrm{Pt}_{x} \mathrm{O}$ system, $\mathrm{Nb}_{5} \mathrm{Ir}_{3} \mathrm{O}$ (i.e., the $x=0.0$ representative of the family) demonstrates the two-gap behavior, which is confirmed by field dependences of the electronic specific heat coefficient and the magnetic penetration depth, as well as by the temperature dependence of the upper critical field [60]. At the same time, the temperature dependences of the specific heat and the superfluid density stay in agreement with the single $s$-wave gap behavior.

The self-consistent two-gap approach, presented here, is used to describe the temperature evolutions of the specific heat $C_{\mathrm{e}}$, the superfluid density $\rho_{\mathrm{s}}$, and the thermodynamic critical field $B_{\mathrm{c}}$. Among them, the specific heat and the superfluid density may not be able to distinguish between the single-gap and two-gap scenario within a certain parameter range. This becomes true for $\mathrm{BeAu}$, studied here, and, most likely, for $\mathrm{Nb}_{5} \mathrm{Ir}_{3} \mathrm{O}$ from Ref. [60]. The deviation of the thermodynamic critical field from the parabolic behavior $D(T)$ reacts, however, quite differently than that of $C_{\mathrm{e}}(T)$ and $\rho_{\mathrm{s}}(T)$. The best way to distinguish between the single- and two-gap behaviors is therefore the simultaneous analysis of $D(T)$ and $C_{\mathrm{e}}(T)$ (as is made in our studies) or $D(T)$ and $\rho_{\mathrm{s}}(T)$ data. Following the results presented in Sec. III A, the analysis of $C_{\mathrm{e}}(T)$ and/or
$\rho_{\mathrm{s}}(T)$ data alone may not lead to correct conclusions. Unfortunately, direct measurements of $B_{\mathrm{c}}(T)$ are quite difficult since the majority of superconducting materials are type-II superconductors.

At the end of this section, we would stress that the two-gap approach presented here is, probably, the simplest one to describe multiband superconductivity. The model considers, in particular, the presence of only two isotropic superconducting energy gaps of $s$-wave symmetry. In a real situation, however, the anisotropy of Fermi surfaces may still lead to a different functional form of $B_{\mathrm{c}}(T)$ or $C_{\mathrm{e}}(T)$. In addition, more than two bands with different energy gaps may intersect the Fermi level.

In $\mathrm{BeAu}$, at least four anisotropic Fermi surfaces were obtained from density functional theory calculations [46-48]. In this respect, the self-consistent two-gap approach is supposed to be used just as the first reliable step for justifying the multiple-gap behaviorx. As soon as the orientational dependences of thermodynamic quantities, such as $B_{\mathrm{c}}, C_{\mathrm{e}}, \lambda, \Delta$, etc., become available, the model could be reconsidered.

\section{CONCLUSIONS}

The temperature evolution of the electronic specific heat $C_{\mathrm{e}}(T)$ and the thermodynamic critical field $B_{\mathrm{c}}(T)$ of the noncentrosymmetric BeAu superconductor were analyzed by means of the single-gap $\alpha$ model and the self-consistent twogap model. Our results confirm that the two-gap approach allows us to describe both thermodynamic quantities with the same set of parameters. The fact that $C_{\mathrm{e}}(T)$ could be interpreted within the single-gap scenario is unique for the $\mathrm{BeAu}$ superconductor. In this particular case, the big and small gap contributions to the electronic specific heat result in behavior which is hardly distinguishable from the single-gap approach. A similar situation was obtained for the temperature dependence of the superfluid density $\rho_{\mathrm{s}}(T) / \rho_{s}(0)$.

Our results imply that in some particular cases, such as the case for BeAu studied here, the measurement of one single thermodynamic quantity may not be sufficient to establish the symmetry of the superconducting order parameter.

\section{ACKNOWLEDGMENTS}

This work was performed at the Swiss Muon Source $(\mathrm{S} \mu \mathrm{S})$, Paul Scherrer Institute (PSI, Switzerland). R.K. acknowledges helpful discussions with R. Lortz. The work of R.G. is supported by the Swiss National Science Foundation (SNF Grant No. 200021-175935).

\section{APPENDIX: THE SINGLE-GAP AND THE TWO-GAP APPROACHES}

This Appendix describes the single-gap and two-gap models, which are traditionally used to analyze temperature dependences of the electronic specific heat $C_{\mathrm{e}}$, the superfluid density $\rho_{\mathrm{s}}$, and the thermodynamic critical field $B_{\mathrm{c}}$. The single-gap approach is based on the model first introduced by Padamsee et al. [34] and is known as the $\alpha$ model. The model was recently reconsidered by Johnston [35]. The two-gap model for analyzing $C_{\mathrm{e}}(T)$ and $\rho_{\mathrm{c}}(T)$ was introduced by Bouquet 
et al. [7], Carrington and Manzano [8], and Fletcher et al. [10] for analyzing the specific heat and the superfluid density data of $\mathrm{MgB}_{2}$. The extension of this model for analyzing the thermodynamic critical field data is given in Ref. [50]. There are two approaches considering different temperature dependences of the big $\left[\Delta_{1}(T)\right]$ and small $\left[\Delta_{2}(T)\right]$ superconducting energy gaps. The simplest, i.e., the two-gap version of the $\alpha$ model, assumes the gaps are described within the weakly coupled BCS approach by following the functional form derived by Mühlschlegel [52]. The more advanced version determines temperature evolutions of the small and big gaps by solving the coupled gap equations self-consistently [41-45].

\section{Single-gap approach}

\section{a. $C_{\mathrm{e}}, \rho_{\mathrm{s}}$, and $B_{\mathrm{c}}$ within the single-gap BCS model}

Within the isotropic $s$-wave gap scenario, the temperature dependences of the electronic specific heat $C_{\mathrm{e}}$, the superfluid density $\rho_{\mathrm{s}}(T) / \rho_{\mathrm{s}}(0)$, and the thermodynamic critical field $B_{\mathrm{c}}(T)$ can be obtained analytically $[3,34,35,50]$ :

$$
\begin{gathered}
\frac{C_{\mathrm{e}}(T)}{\gamma_{\mathrm{n}} T_{\mathrm{c}}}=\frac{6}{\pi^{2} k_{\mathrm{B}}^{3} T_{\mathrm{c}} T^{2}} \int_{0}^{\infty} f(1-f) \\
\times\left[\epsilon^{2}+\Delta(T)^{2}-\frac{T \partial \Delta(T)^{2}}{2 \partial T}\right] d \epsilon, \\
\frac{\rho_{\mathrm{s}}(T)}{\rho_{\mathrm{s}}(0)}=1-\frac{1}{2 T} \int_{0}^{\infty} \cosh ^{-2}\left[\frac{\sqrt{\epsilon^{2}+\Delta(T)^{2}}}{2 T}\right] d \epsilon,
\end{gathered}
$$

and

$$
\frac{B_{\mathrm{c}}^{2}(T)}{\gamma_{\mathrm{n}} T_{\mathrm{c}}^{2}}=8 \pi \frac{F_{\mathrm{n}}-F_{\mathrm{s}}}{\gamma_{\mathrm{n}} T_{\mathrm{c}}^{2}}
$$

Here, $f=\left\{1+\exp \left[\sqrt{\epsilon^{2}+\Delta(T)^{2}} / k_{\mathrm{B}} T\right]\right\}^{-1}$ is the Fermi function, and $\gamma_{\mathrm{n}}$ is the normal-state electronic specific heat coefficient (Sommerfeld constant). $F_{\mathrm{n}}$ and $F_{\mathrm{s}}$ are the normaland superconducting-state free energies given by $[35,50]$

$$
\frac{F_{\mathrm{n}}}{\gamma_{\mathrm{n}} T_{\mathrm{c}}^{2}}=-\frac{T^{2}}{2 T_{\mathrm{c}}^{2}}
$$

and

$$
\begin{aligned}
& \frac{F_{\mathrm{s}}[T, \Delta(T)]}{\gamma_{\mathrm{n}} T_{\mathrm{c}}^{2}} \\
& \quad=-\frac{3}{\pi^{2} k_{\mathrm{B}}^{2} T_{\mathrm{c}}^{2}}\left[\frac{\Delta(T)^{2}}{4}+\int_{0}^{\infty} f \frac{2 \epsilon^{2}+\Delta(T)^{2}}{\sqrt{\epsilon^{2}+\Delta(T)^{2}}} d \epsilon\right] .
\end{aligned}
$$

Note that Eqs. (A1), (A3), (A4), and (A5) are expressed in cgs units [35].

\section{b. Single-gap $\alpha$ model}

Originally, the $\alpha$ model was adapted from the single-band BCS theory of superconductivity in order to explain deviations of the temperature behavior of the electronic specific heat and the thermodynamic critical field from the weakly coupled BCS prediction [34,35]. The model assumes that the temperature dependence of the normalized superconducting

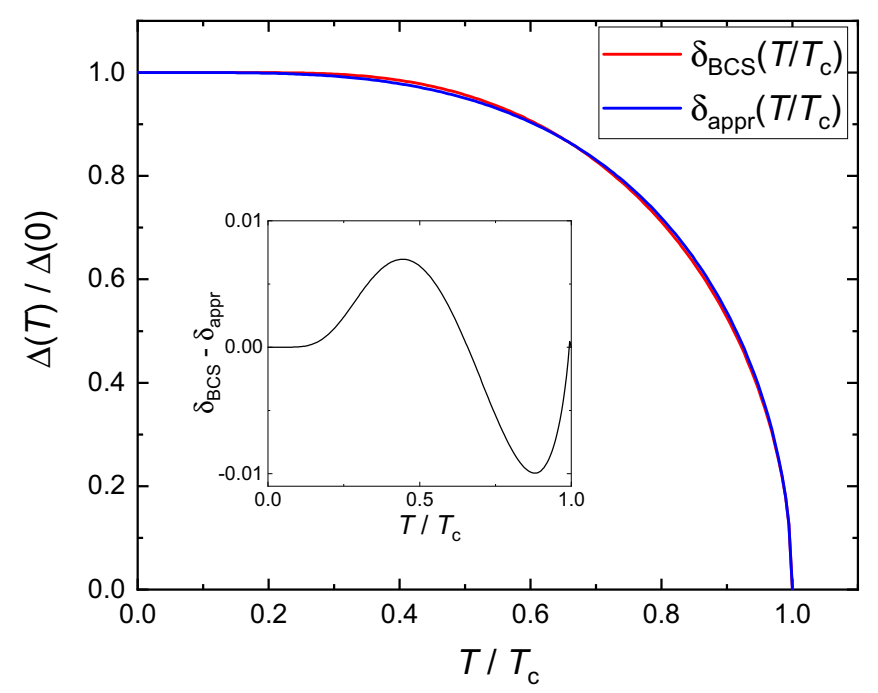

FIG. 7. Normalized BCS energy gap $\delta_{\mathrm{BCS}}\left(T / T_{\mathrm{c}}\right)=$ $\Delta_{\mathrm{BCS}}\left(T / T_{\mathrm{c}}\right) / \Delta_{\mathrm{BCS}}(0)$, obtained by solving the self-consistent single-gap equation (A8) (red curve) and the approximated gap calculated by means of Eq. (A9) (blue curve). The inset shows the difference $\delta_{\mathrm{BCS}}\left(T / T_{\mathrm{c}}\right)-\delta_{\mathrm{appr}}\left(T / T_{\mathrm{c}}\right)$ between the BCS and the approximated solutions.

energy gap

$$
\delta_{\mathrm{BCS}}(T)=\frac{\Delta(T)}{\Delta(0)}=\frac{\Delta_{\mathrm{BCS}}(T)}{\Delta_{\mathrm{BCS}}(0)}
$$

$[\Delta(0)$ is the zero-temperature value of the gap] is the same as that for BCS theory [52]. The parameter

$$
\alpha=\frac{\Delta(0)}{k_{\mathrm{B}} T_{\mathrm{c}}}
$$

is further introduced in order to account for the deviation from the weakly coupled BCS prediction $\alpha_{\mathrm{BCS}} \simeq 1.764$.

The BCS temperature dependence of the gap is obtained by solving the self-consistent gap equation $[3,35]$ :

$$
1=N V \int_{0}^{\omega_{\mathrm{D}}} \frac{1}{\sqrt{\epsilon^{2}+\Delta(T)^{2}}} \tanh \frac{\epsilon^{2}+\Delta(T)^{2}}{2 k_{\mathrm{B}} T} d \epsilon .
$$

Here, $\omega_{\mathrm{D}}$ is the Debye frequency, $N$ is the normal-state electronic density of states at the Fermi level, and $V$ is the electron-phonon interaction potential. The temperature dependence of the normalized BCS gap is normally approximated by [8]

$$
\delta_{\text {appr }}(T)=\tanh \left\{1.82\left[1.018\left(T_{\mathrm{c}} / T-1\right)\right]^{0.51}\right\},
$$

which results in less than $1 \%$ deviation from the exact solution of Eq. (A8).

Figure 7 compares the exact solution of Eq. (A8) with the results of Eq. (A9). The deviation of the approximated gap function $\delta_{\text {appr }}\left(T / T_{c}\right)$ from $\delta_{\mathrm{BCS}}\left(T / T_{\mathrm{c}}\right)$ is given in the inset.

\section{Two-gap approach}

\section{a. $C_{\mathrm{e}}, \rho_{\mathrm{s}}$, and $B_{\mathrm{c}}$ within the two-gap model}

Following Refs. [7-9,11-14,41,44,50], the temperature evolutions of $C_{\mathrm{e}}, \rho_{\mathrm{s}}$, and $B_{\mathrm{c}}$ within the two-gap approach can 
be obtained as

$$
\begin{aligned}
\frac{C_{\mathrm{e}}(T)}{\gamma_{\mathrm{n}} T_{\mathrm{c}}} & =\frac{C_{\mathrm{e} 1}(T)+C_{\mathrm{e} 2}(T)}{\gamma_{\mathrm{n}} T_{\mathrm{c}}} \\
& =w_{C_{\mathrm{e}}} \frac{C_{\mathrm{e} 1}(T)}{\gamma_{\mathrm{n} 1} T_{\mathrm{c}}}+\left(1-w_{C_{\mathrm{e}}}\right) \frac{C_{\mathrm{e} 2}(T)}{\gamma_{\mathrm{n} 2} T_{\mathrm{c}}}, \\
\frac{\rho_{\mathrm{s}}(T)}{\rho_{\mathrm{s}}(0)} & =\frac{\rho_{\mathrm{s} 1}(T)+\rho_{\mathrm{s} 2}(T)}{\rho_{\mathrm{s}}(0)} \\
& =w_{\rho} \frac{\rho_{\mathrm{s} 1}(T)}{\rho_{\mathrm{s} 1}(0)}+\left(1-w_{\rho}\right) \frac{\rho_{\mathrm{s} 2}(T)}{\rho_{\mathrm{s} 2}(0)},
\end{aligned}
$$

and

$$
\begin{aligned}
\frac{B_{\mathrm{c}}^{2}(T)}{\gamma_{\mathrm{n}} T_{\mathrm{c}}^{2}} & =8 \pi \frac{F_{\mathrm{n}}-F_{\mathrm{s} 1}-F_{\mathrm{s} 2}}{\gamma_{\mathrm{n}} T_{\mathrm{c}}^{2}} \\
& =8 \pi\left[\frac{F_{\mathrm{n}}}{\gamma_{\mathrm{n}} T_{\mathrm{c}}^{2}}-w_{F_{\mathrm{s}}} \frac{F_{\mathrm{s} 1}}{\gamma_{\mathrm{n} 1} T_{\mathrm{c}}^{2}}-\left(1-w_{F_{\mathrm{s}}}\right) \frac{F_{\mathrm{s} 2}}{\gamma_{\mathrm{n} 2} T_{\mathrm{c}}^{2}}\right] .
\end{aligned}
$$

Here, 1 and 2 are gap indices. $w_{C_{\mathrm{e}}}, w_{\rho}$, and $w_{F_{\mathrm{s}}}$ are the contributions of the first gap to the resulting electronic specific heat, the superfluid density, and the superconducting free energy, respectively. $\gamma_{\mathrm{n}}=\gamma_{\mathrm{n} 1}+\gamma_{\mathrm{n} 2}$. Note that in accordance with Eqs. (A10) and (A12)

$$
w_{C_{\mathrm{e}}} \equiv w_{F_{\mathrm{s}}}=\frac{\gamma_{n 1}}{\gamma_{n 1}+\gamma_{n 2}} .
$$

The analytical equation for $w_{\rho}$ was calculated by Kogan et al. [41], within the framework of the self-consistent two-gap model. $w_{\rho}$ was found to depend on the partial densities of states and the average Fermi velocities of the first and second bands, respectively. In general, $w_{\rho} \neq w_{C_{\mathrm{e}}} \equiv w_{F_{\mathrm{s}}}$.

\section{b. Self-consistent two-gap model}

The self-consistent two-gap model was introduced shortly after the formulation of the BCS theory by Suhl et al. [61] and Moskalenko [62] in order to account for a more complex Fermi surface topology than the one introduced by BCS weakly coupled theory. Extensions of this approach were suggested shortly afterward [63]. The important clue of these extensions to superconductivity is an interband pair scattering potential, which leads to enhanced pair scattering via exchange through an additional channel. The revised version of the model for two isotropic $s$-wave gaps was recently reconsidered in a series of publications by Kogan et al. [41,42] and Bussmann-Holder et al. [43-45]. Here, the approach developed in Refs. [43-45] is described.

Following Refs. [43-45,50,64,65], within the two-gap approach, the coupled $s$-wave gap equations are described as

$$
\Delta_{i}(T)=\sum_{j=1}^{2} \int_{0}^{\omega_{D_{j}}} \frac{N_{j} V_{i j} \Delta_{j}(T)}{\sqrt{\epsilon^{2}+\Delta_{j}^{2}(T)}} \tanh \frac{\sqrt{\epsilon^{2}+\Delta_{j}^{2}(T)}}{2 k_{\mathrm{B}} T} d \epsilon .
$$

Here, $N_{i}$ is the partial density of states for the $i$ th band at the Fermi level $\left(N_{1}+N_{2}=1\right) ; V_{i i}$ and $V_{i j \neq i}$ are the intraband and interband interaction potentials, respectively. For simplicity, one normally assumes that the Debye frequency $\omega_{\mathrm{D}}$ is the same for both bands $\left(\omega_{\mathrm{D} 1}=\omega_{\mathrm{D} 2}=\omega_{\mathrm{D}}\right)[41,44,50,64,65]$.

The system of self-consistent coupled gap equations [Eq. (A14)] might be solved for certain values of the intraband $\left(V_{11}, V_{22}\right)$ and interband $\left(V_{12}, V_{21}\right)$ coupling potentials and the Debye frequency $\left(\omega_{\mathrm{D}}\right)$, which results in temperature dependences of the big $\Delta_{1}(T)$ and small $\Delta_{2}(T)$ superconducting energy gaps. The respective $\Delta_{1}(T)$ and $\Delta_{2}(T)$ are further substituted into Eqs. (A10), (A11), and (A12) [with the individual components described by Eqs. (A1), (A2), (A4), and (A5)], and the temperature dependences of $C_{\mathrm{e}}(T), \rho_{\mathrm{s}}(T)$, and $B_{\mathrm{c}}(T)$ are obtained.
[1] D. Shoenberg, Superconductivity (Cambridge University Press, Cambridge, 1952).

[2] Superconductivity (in Two Volumes), edited by R. D. Parks (Dekker, New York, 1969).

[3] M. Tinkham, Introduction to Superconductivity (Krieger, Malabar, FL, 1975).

[4] D. R. Tilley and J. Tilley, Superfluids and Superconductivity, 3rd ed. (IOP Publishing, Bristol, 1990).

[5] P. G. de Gennes, Superconductivity in Metals and Alloys (Perseus, Cambridge, MA, 1999).

[6] C. P. Poole, R. Prozorov, H. A. Farach, and R. J. Creswick Superconductivity, 3rd ed. (Elsevier, Amsterdam, 2014).

[7] F. Bouquet, Y. Wang, R. A. Fisher, D. G. Hinks, J. D. Jorgensen, A. Junod, and N. E. Phillips, Europhys. Lett. 56, 856 (2001).

[8] A. Carrington and F. Manzano, Phys. C (Amsterdam, Neth.) 385, 205 (2003).

[9] V. Guritanu, W. Goldacker, F. Bouquet, Y. Wang, R. Lortz, G. Goll, and A. Junod, Phys. Rev. B 70, 184526 (2004).
[10] J. D. Fletcher, A. Carrington, O. J. Taylor, S. M. Kazakov, and J. Karpinski, Phys. Rev. Lett. 95, 097005 (2005).

[11] R. Prozorov and R. W. Giannetta, Supercond. Sci. Technol. 19, R41 (2006).

[12] R. Khasanov, A. Shengelaya, A. Maisuradze, F. La Mattina, A. Bussmann-Holder, H. Keller, and K. A. Müller, Phys. Rev. Lett. 98, 057007 (2007).

[13] R. Khasanov, S. Strässle, D. Di Castro, T. Masui, S. Miyasaka, S. Tajima, A. Bussmann-Holder, and H. Keller, Phys. Rev. Lett. 99, 237601 (2007).

[14] R. Khasanov, A. Shengelaya, J. Karpinski, A. BussmannHolder, H. Keller, and K. A. Müller, J. Supercond. Novel Magn. 21, 81 (2008).

[15] Y. Nakajima, T. Nakagawa, T. Tamegai, and H. Harima, Phys. Rev. Lett. 100, 157001 (2008).

[16] R. Khasanov, T. Kondo, S. Strässle, D. O. G. Heron, A. Kaminski, H. Keller, S. L. Lee, and T. Takeuchi, Phys. Rev. Lett. 101, 227002 (2008). 
[17] R. T. Gordon, M. D. Vannette, C. Martin, Y. Nakajima, T. Tamegai, and R. Prozorov, Phys. Rev. B 78, 024514 (2008).

[18] R. Khasanov, T. Kondo, S. Strässle, D. O. G. Heron, A. Kaminski, H. Keller, S. L. Lee, and T. Takeuchi, Phys. Rev. B 79, 180507(R) (2009).

[19] J. Chen, L. Jiao, J. L. Zhang, Y. Chen, L. Yang, M. Nicklas, F. Steglich, and H. Q. Yuan, New J. Phys. 15, 053005 (2013).

[20] R. Khasanov, H. Zhou, A. Amato, Z. Guguchia, E. Morenzoni, X. Dong, G. Zhang, and Z. Zhao, Phys. Rev. B 93, 224512 (2016).

[21] V. Yu. Verchenko, R. Khasanov, Z. Guguchia, A. A. Tsirlin, and A. V. Shevelkov, Phys. Rev. B 96, 134504 (2017).

[22] I. Bonalde, R. L. Ribeiro, W. Brämer-Escamilla, G. Mu, and H. H. Wen, Phys. Rev. B 79, 052506 (2009).

[23] M. Mondal, B. Joshi, S. Kumar, A. Kamlapure, S. C. Ganguli, A. Thamizhavel, S. S. Mandal, S. Ramakrishnan, and P. Raychaudhuri, Phys. Rev. B. 86, 094520 (2012).

[24] C. Cirillo, R. Fittipaldi, M. Smidman, G. Carapella, C. Attanasio, A. Vecchione, R. P. Singh, M. R. Lees, G. Balakrishnan, and M. Cuoco, Phys. Rev. B 91, 134508 (2015).

[25] M. Smidman, M. B. Salamon, H. Q. Yuan, and D. F. Agterberg, Rep. Prog. Phys. 80, 036501 (2017).

[26] Y. Nakayama and T. Muranaka, Inorg. Chem. 58, 12733 (2019).

[27] Y. Singh, C. Martin, S. L. Bud'ko, A. Ellern, R. Prozorov, and D. C. Johnston, Phys. Rev. B 82, 144532 (2010).

[28] R. Khasanov, T. Kondo, M. Bendele, Y. Hamaya, A. Kaminski, S. L. Lee, S. J. Ray, and T. Takeuchi, Phys. Rev. B 82, 020511(R) (2010).

[29] H. Kim, M. A. Tanatar, Y. J. Song, Y. S. Kwon, and R. Prozorov, Phys. Rev. B 83, 100502(R) (2011).

[30] R. Khasanov, W. R. Meier, Y. Wu, D. Mou, S. L. Bud'ko, I. Eremin, H. Luetkens, A. Kaminski, P. C. Canfield, and A. Amato, Phys. Rev. B 97, 140503(R) (2018).

[31] R. Khasanov, W. R. Meier, S. L. Bud'ko, H. Luetkens, P. C. Canfield, and A. Amato, Phys. Rev. B 99, 140507(R) (2019).

[32] H. Kim, K. Cho, M. A. Tanatar, V. Taufour, S. K. Kim, S. L. Bud'ko, P. C. Canfield, V. G. Kogan, and R. Prozorov, Symmetry 11, 1012 (2019).

[33] R. Khasanov, H. Luetkens, E. Morenzoni, G. Simutis, S. Schönecker, A. Östlin, L. Chioncel, and A. Amato, Phys. Rev. B 98, 140504(R) (2018).

[34] H. Padamsee, J. E. Neighbor, and C. A. Shiffman, J. Low Temp. Phys. 12, 387 (1973).

[35] D. C. Johnston, Supercond. Sci. Technol. 26, 115011 (2013).

[36] R. Khasanov, M. M. Radonjić, H. Luetkens, E. Morenzoni, G. Simutis, S. Schönecker, W. H. Appelt, A. Östlin, L. Chioncel, and A. Amato, Phys. Rev. B 99, 174506 (2019).

[37] R. Karl, F. Burri, A. Amato, M. Donegà, S. Gvasaliya, H. Luetkens, E. Morenzoni, and R. Khasanov, Phys. Rev. B 99, 184515 (2019).

[38] R. Khasanov, H. Luetkens, A. Amato, and E. Morenzoni, Phys. Rev. B 101, 054504 (2020).

[39] J. P. Carbotte, Rev. Mod. Phys. 62, 1027 (1990).

[40] F. Marsiglio and J. P. Carbotte, Electron-Phonon Superconductivity, in Superconductivity, edited by K. H. Bennemann and J. B. Ketterson (Springer, Berlin, 2008), pp. 73-162.

[41] V. G. Kogan, C. Martin, and R. Prozorov, Phys. Rev. B 80, 014507 (2009).
[42] V. G. Kogan and R. Prozorov, Phys. Rev. B 93, 224515 (2016).

[43] A. Bussmann-Holder, R. Micnas, and A. R. Bishop, Eur. Phys. J. B. 37, 345 (2004).

[44] A. Bussmann-Holder, arXiv:0909.3603.

[45] A. Bussmann-Holder, H. Keller, A. Simon, and A. Bianconi, Condens. Matter 4, 91 (2019).

[46] D. Rebar, Ph.D. dissertation, Louisiana State University, 2015.

[47] A. Amon, E. Svanidze, R. Cardoso-Gil, M. N. Wilson, H. Rosner, M. Bobnar, W. Schnelle, J. W. Lynn, R. Gumeniuk, C. Hennig, G. M. Luke, H. Borrmann, A. Leithe-Jasper, and Yu. Grin, Phys. Rev. B 97, 014501 (2018).

[48] D. J. Rebar, S. M. Birnbaum, J. Singleton, M. Khan, J. C. Ball, P. W. Adams, J. Y. Chan, D. P. Young, D. A. Browne, and J. F. Di Tusa, Phys. Rev. B 99, 094517 (2019).

[49] D. Singh, A. D. Hillier, and R. P. Singh, Phys. Rev. B 99, 134509 (2019).

[50] R. Khasanov, R. Gupta, D. Das, A. Amon, A. Leithe-Jasper, and E. Svanidze, Phys. Rev. Research 2, 023142 (2020).

[51] J. Beare, M. Nugent, M. N. Wilson, Y. Cai, T. J. S. Munsie, A. Amon, A. Leithe-Jasper, Z. Gong, S. L. Guo, Z. Guguchia, Y. Grin, Y. J. Uemura, E. Svanidze, and G. M. Luke, Phys. Rev. B 99, 134510 (2019).

[52] B. Mühlschlegel, Z. Phys. 155, 313 (1959).

[53] The samples used in the $C_{\mathrm{e}}(T)$ and $B_{\mathrm{c}}(T)$ experiments came from the same batch, but they were not identical. A tiny $T_{\mathrm{c}}$ deviation from one sample piece to another may easily take place. In our case such a deviation accounts for a superconducting transition temperature difference of less than $\sim 60 \mathrm{mK}$, i.e., less than $1.7 \%$.

[54] A. A. Golubov, J. Kortus, O. V. Dolgov, O. Jepsen, Y. Kong, O. K. Andersen, B. J. Gibson, K. Ahn, and R. K. Kremer, J. Phys.: Condens. Matter 14, 1353 (2002).

[55] A. Floris, G. Profeta, N. N. Lathiotakis, M. Lüders, M. A. L. Marques, C. Franchini, E. K. U. Gross, A. Continenza, and S. Massidda, Phys. Rev. Lett. 94, 037004 (2005).

[56] K. Chen, Y. Cui, Q. Li, C. G. Zhuang, Z. K. Liu, and X. X. Xi, Appl. Phys. Lett. 93, 012502 (2008).

[57] H. Luetkens, H.-H. Klauss, R. Khasanov, A. Amato, R. Klingeler, I. Hellmann, N. Leps, A. Kondrat, C. Hess, A. Köhler, G. Behr, J. Werner, and B. Büchner, Phys. Rev. Lett. 101, 097009 (2008).

[58] R. S. Gonnelli, D. Daghero, M. Tortello, G. A. Ummarino, V. A. Stepanov, R. K. Kremer, J. S. Kim, N. D. Zhigadlo, and J. Karpinski, Phys. C (Amsterdam, Neth.) 469, 512 (2009).

[59] R. Khasanov, A. Amato, P. K. Biswas, H. Luetkens, N. D Zhigadlo, and B. Batlogg, Phys. Rev. B 90, 140507(R) (2014).

[60] Y. Xu, S. Jöhr, L. Das, J. Kitagawa, M. Medarde, T. Shiroka, J. Chang, and T. Shang, Phys. Rev. B 101, 134513 (2020).

[61] H. Suhl, B. T. Matthias, and L. R. Walker, Phys. Rev. Lett. 3, 552 (1959).

[62] V. A. Moskalenko, Fiz. Metal Metalloved. 8, 503 (1959).

[63] B. Geilikman, R. Zaitsev, and V. Z. Kresin, Sov. Phys. Solid State 9, 524 (1966).

[64] R. Khasanov, M. Bendele, A. Amato, K. Conder, H. Keller, H.H. Klauss, H. Luetkens, and E. Pomjakushina, Phys. Rev. Lett. 104, 087004 (2010).

[65] R. Gupta, A. Maisuradze, N. D. Zhigadlo, H. Luetkens, A. Amato, and R. Khasanov, Front. Phys. 8, 2 (2020). 